\title{
Magnetic Contrast at Spin-Flip Excitations: An Advanced X-Ray Spectroscopy Tool to Study Magnetic-Ordering
}

\author{
Hebatalla Elnaggar, ${ }^{* \dagger \odot}$ Ru-Pan Wang, ${ }^{\dagger}$ Sara Lafuerza, ${ }^{\ddagger}$ Eugenio Paris, ${ }^{\text {II }}$ Yi Tseng, ${ }^{\text {If }}$ Daniel McNally, ${ }^{\text {II }}$ \\ Alexander Komarek, ${ }^{\S}$ Maurits Haverkort, ${ }^{\|}$Marcin Sikora, ${ }^{\perp}$ Thorsten Schmitt, ${ }^{\text {I }}$ \\ and Frank M. F. de Groot*ं† \\ ${ }^{\dagger}$ Debye Institute for Nanomaterials Science, Utrecht University, Universiteitsweg 99, 3584 CA Utrecht, The Netherlands \\ "European Synchrotron Radiation Facility, 71 Avenue des Martyrs, CS 40220, 38043 Grenoble, France \\ "Photon Science Division, Paul Scherrer Institut, Forschungsstrasse 111, 5232 Villigen PSI, Switzerland \\ ${ }^{\S}$ Max-Planck-Institute for Chemical Physics of Solids, Nöthnitzer Str. 40, 01187 Dresden, Germany \\ "Institut für Theoritiche Physik, Universität Heidelberg, Philosophenweg 19, 69120 Heidelberg, Germany \\ ${ }^{\perp}$ Academic Centre for Materials and Nanotechnology, AGH University of Science and Technology, Mickiewicza 30, 30-059 Krakow,
} Poland

Supporting Information

ABSTRACT: The determination of the local orientation and magnitude of the magnetization in spin textures plays a pivotal role in understanding and harnessing magnetic properties for technological applications. Here, we show that by employing the polarization dependence of resonant inelastic X-ray scattering (RIXS), we can directly probe the spin ordering with chemical and site selectivity. Applied on the prototypical ferrimagnetic mixed-valence system, magnetite $\left(\left[\mathrm{Fe}^{3+}\right]_{\mathrm{A}}\left[\mathrm{Fe}^{3+}, \mathrm{Fe}^{2+}\right]_{\mathrm{B}} \mathrm{O}_{4}\right)$, we can distinguish spin-flip excitations at the $\mathrm{A}$ and $\mathrm{B}$ antiferromagnetically coupled $\mathrm{Fe}^{3+}$ sublattices and quantify the exchange field. Furthermore, it is possible to determine the orbital contribution to the magnetic moment from detailed angular dependence measurements. RIXS dichroism measurements performed at spin-flip excitations with nanometer spatial resolution will offer a powerful mapping contrast suitable for the characterization of magnetic ordering at interfaces and engineered spin textures.

KEYWORDS: site-selective spin excitations, resonant inelastic X-ray scattering, magnetite, spin-flip dichroism, spin-states mapping

\section{INTRODUCTION}

Magnetic phenomena associated with spin-orbit coupling, the Dzyaloshinskii-Moriya interaction, exchange bias, and magnetic anisotropy have led to a wide variety of emergent spin textures. In particular, many exotic spin states have been realized at interfaces of films, multilayers, and superstructures, ranging from emergent magnetism at thin film carbon-based magnets ${ }^{1}$ and cycloidal spin spirals at $\mathrm{Fe} / \mathrm{W}$ bilayers ${ }^{2}$ to topologically protected magnetic skyrmions in $\mathrm{Fe} / \mathrm{Ir}$-based films. ${ }^{3-5}$ Understanding and designing the emergent magnetic behavior by tuning such interactions is crucial for many technological applications such as information storage, spin transport, and sensing technology. However, for this progress to take place, new and advanced characterization tools of spin states are required.

A particularly challenging topic to investigate is that of transition-metal oxide interfaces where significant changes to the atomic and electronic structure are commonly induced. This can lead to interfacial magnetism and transport effects that are not a simple interpolation of the properties of the adjoined materials. An example of such intriguing systems is magnetite $\left(\mathrm{Fe}_{3} \mathrm{O}_{4}\right)$ because of its half metallicity and strong spin polarization at the Fermi level. $\mathrm{Fe}_{3} \mathrm{O}_{4}$ can be interfaced with oxide electrodes, leading to interesting effects, such as spin-to-charge conversion, spin-dependent transport $^{6}$ (spin filter, spin valve, spin injection, and magnetic tunnel junctions), and the spin Seebeck effect. ${ }^{7}$ Moreover, $\mathrm{Fe}_{3} \mathrm{O}_{4}$ exhibits a metal-to-insulator transition at $\sim 125 \mathrm{~K}$, which results in a spontaneous change of both the lattice symmetry and the electric conductivity. This drastic switch of conductivity was found to occur in the picosecond timescale, ${ }^{8,9}$ opening novel avenues for designing fast electronics. Verwey proposed that at $T_{\mathrm{V}}$, a charge order-to-disorder transition takes place. ${ }^{10}$ Ever since this formulation, extensive efforts have been exerted to finding evidence for the proposed charge ordering (and later

Received: June 11, 2019

Accepted: September 9, 2019

Published: September 9, 2019 

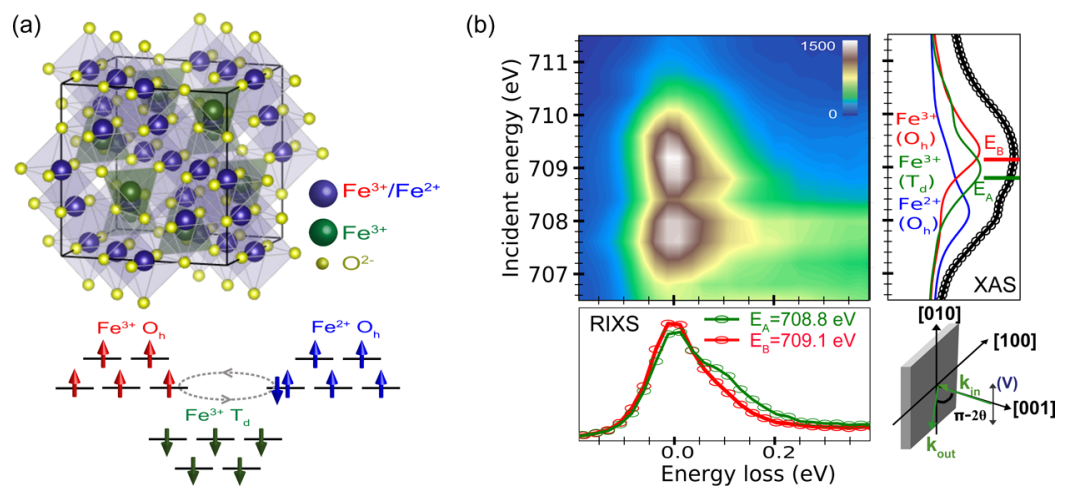

Figure 1. Fe 2p3d RIXS measurements in $\mathrm{Fe}_{3} \mathrm{O}_{4}$. (a) Unit cell of $\mathrm{Fe}_{3} \mathrm{O}_{4}$ and the magnetic coupling between the Fe sites are illustrated. Octahedral $\left(\mathrm{O}_{\mathrm{h}}\right) \mathrm{Fe}^{3+}$ and $\mathrm{Fe}^{2+} \mathrm{B}$ sites are antiferromagnetically coupled to the tetrahedral $\left(\mathrm{T}_{\mathrm{d}}\right) \mathrm{Fe}^{3+}$ A site. The gray arrow represents the rapid hopping of the spin-down electron between the Fe B sites. (b) $\mathrm{Fe}_{3}$ RIXS map of $\mathrm{Fe}_{3} \mathrm{O}_{4}$. The $L_{3}$ XAS signal is shown on the right. The theoretical contributions of the three Fe ions to the XAS signal are plotted. Two incident energies were chosen for the high-resolution RIXS measurements, namely, $E_{\mathrm{A}}=708.8$ and $E_{\mathrm{B}}=709.1 \mathrm{eV}$ as shown at the bottom panel. A sketch of the scattering geometry used is shown in the right bottom corner where $V$ is the vertical incident polarization, $\mathbf{k}_{\text {in }}$ and $\mathbf{k}_{\text {out }}$ are the incident and scattered wave vectors, respectively, and $2 \theta$ is set to $130^{\circ}$.

on, orbital ordering) and the accompanying distortion at the low-temperature phase. ${ }^{11-18}$

To understand and design tailored functionality in such a complex system, it is desirable to directly probe and quantify the charge, spin, and orbital degrees of freedom with chemical and site selectivity. This remains a task beyond standard techniques such as scanning transmission electron microscopy (STEM), magnetic optical Kerr effect (MOKE), X-ray and neutron non-resonant diffraction, and reflectivity measurements. Although spin-polarized X-ray photoemission (SP-XPS) and electron energy loss spectroscopy (EELS) can potentially provide valuable information, they are surface-sensitive (a few monolayers) or require sophisticated sample treatment. Moreover, the popular resonant X-ray magnetic diffraction technique, ${ }^{19,20}$ where the different scattering amplitudes interfere coherently offering both the short-range order sensitivity of absorption and the long-range order sensitivity of diffraction, faces a challenge to satisfy both the energy condition for resonance and the momentum condition for the appropriate diffraction peak simultaneously.

Resonant inelastic X-ray scattering (RIXS) is a photon-in, photon-out technique where the incident energy is tuned such that it resonates with a specific element exciting an electron out of its core shells into an empty electronic state. The resonance provides chemical selectivity to a certain atom and enhances the inelastic scattering cross section, offering a unique way to probe charge, spin, and orbital degrees of freedom. ${ }^{21,22}$ The highly excited atom contains a localized hole in the core that exists for a few picoseconds. This intermediate state can decay emitting photons from which the energies are measured to determine the energy deposited in the material and construct a RIXS spectrum. Under the appropriate settings of energy, momenta, and photon polarization, the energy transferred to the atom can change the direction of an electron spin at the local absorbing atom (or multispins), an excitation referred to as a spin-flip excitation. ${ }^{23-27}$ As the RIXS process is coherent and conserves momentum, spin-flip excitations can propagate through the lattice (i.e., the local spin-flip response function represents the magnon density of states). At $L_{2,3}$-edges of transition metals, the spin-flip process gains intensity due to the strong spin-orbit coupling at the intermediate state. ${ }^{23}$ The energy required to change the direction of the spin is proportional to the strength of the exchange field and can be used to determine the exchange interaction using the linearized spin wave theory for a Heisenberg model on the $\mathrm{Fe}_{3} \mathrm{O}_{4}$ lattice with nearest-neighbor interactions.

Spin-flip excitations are not only sensitive to the atomic spin state, but they also probe the long-range order in a magnetic system. Collective magnetic excitations can be probed by momentum-resolved RIXS measurements, offering a valuable alternative to neutron measurements. ${ }^{28-31}$ As core electrons do not disperse, the effective RIXS operator becomes local (depending on polarization, experimental geometry, and the excitation mechanism of the local absorbing atom) multiplied by an appropriate spin susceptibility, which acquires a strong momentum dependence for systems with interatomic spinspin interactions. ${ }^{25,28}$ Through studying the effect of the incident X-ray polarization on the spin-flip excitations, it is possible to unambiguously identify these excitations among other low energy loss excitations (such as phonons or crystalfield excitations) and reconstruct the orientation of the magnetic moment. Two types of dichroic signals are particularly important to measure at the spin-flip excitation for building a comprehensive picture, namely, RIXS magnetic circular dichroism (RIXS-MCD) and RIXS magnetic linear dichroism (RIXS-MLD). Magnetic selectivity imposed by the incident polarization handedness, referred to as RIXS$\mathrm{MCD},{ }^{32,33}$ enables the quantification of magnetic moments along the incident beam direction. The dependence of the intensity at the spin-flip excitation on the angle between the linearly polarized X-rays and the magnetic moment (with respect to the low-symmetry axis of the crystal) is referred to as RIXS-MLD. The RIXS-MLD signal can be used to determine the magnetic moment perpendicular to the incident beam. We note that it is also possible to study the polarization dependence in the emission channel to identify magnetic excitations using polarization analyzers; however, this is experimentally more difficult due to their low efficiency. ${ }^{34,35}$

In this work, we employed a combination of high-resolution Fe 2p3d RIXS-MCD and RIXS-MLD measurements in a magnetite $\left(\mathrm{Fe}_{3} \mathrm{O}_{4}\right)$ single crystal to probe low-energy spin-flip excitations and demonstrate the capabilities of RIXS as an advanced magnetic probe. $\mathrm{Fe}_{3} \mathrm{O}_{4}$ serves as an adequate model system: it is a mixed-valence strongly correlated system with two antiferromagnetically coupled sublattices. Above the metal-to-insulator transition (the Verwey transition at $T_{\mathrm{V}} \approx$ 
$\left.125 \mathrm{~K}^{10}\right), \mathrm{Fe}_{3} \mathrm{O}_{4}$ has a cubic inverse spinel crystal structure containing two different $\mathrm{Fe}$ sites. $\mathrm{Fe}^{3+}$ ions reside in tetrahedral $\left(\mathrm{T}_{\mathrm{d}}\right)$ coordinated sites (A sites), while both $\mathrm{Fe}^{2+}$ and $\mathrm{Fe}^{3+}$ ions are in octahedral $\left(\mathrm{O}_{\mathrm{h}}\right)$ coordinated sites (B sites). The A and B sublattices are antiferromagnetically coupled, while the Fe ions at the same sublattice are ferromagnetically coupled (refer to Figure 1a).

By harnessing the magnetic contrast offered by RIXS-MCD, we identified spin-flip excitations and quantified the effective exchange interaction at each of the sublattices. Mapping the polarization dependence of the spin-flip excitation revealed the sensitivity of the measurement to in- and out-of-plane magnetic ordering. The combination of RIXS-MCD and RIXS-MLD can be used to characterize complex magnetic structures including non-collinear and chiral ordering. Guided by theory, we demonstrated that it is possible to identify orbital contributions to the magnetic moment and proved that the magnetic orbital moment at the Fe A sites is quenched. The methodology developed here has far-reaching implications especially when spin-flip dichroism measurements are performed with spatial resolution to image magnetic interfaces.

\section{METHODS}

2.1. Sample Characterization and Treatment. We studied a high-quality (001) $\mathrm{Fe}_{3} \mathrm{O}_{4}$ single crystal grown at the Max Planck Institute for Chemical Physics of Solids in Dresden. The stoichiometry of the single crystal was examined by performing temperature-dependent magnetic measurements. A sharp transition was observed at $125 \mathrm{~K}$ as expected for a highly stoichiometric sample (see Figure S1 of the Supporting Information). The crystallinity and homogeneity of the sample were assessed by measuring the Darwin width of the (008) reflection across the sample at beamline ID28 of the European Synchrotron Radiation Facility (Figure S3). In order to clean and remove damaged surface layers, we exposed the surface to boiling concentrated $\mathrm{HCl}$ for $\sim 30 \mathrm{~s}$ before the measurement.

2.2. XAS and RIXS Measurements. $\mathrm{Fe} L_{2,3}$-edge $\mathrm{X}$-ray absorption spectroscopy (XAS) and resonant inelastic X-ray scattering (RIXS) measurements were carried out at the ADRESS beamline of the Swiss Light Source at the Paul Scherrer Institut, Switzerland. ${ }^{36}$ The high-brilliance X-ray beam was monochromatized using a plane grating with a constant groove density of $800 \mathrm{~mm}^{-1}$ and focused down to a spot of $\leq 4 \mu \mathrm{m} \times 55 \mu \mathrm{m}$ size at the sample position using an elliptical refocusing mirror. All measurements were performed in a normal incidence scattering geometry, that is, with the incoming beam impinging at an angle of $90^{\circ}$ with respect to the sample surface. The scattering angle was set to $2 \theta=130^{\circ}$, which is the largest experimental scattering angle possible at the beamline to minimize the elastic scattering angular dependence (see Figure S12). The energy analysis of the emitted radiation was performed using a variable-line-spacing spherical grating, with an average groove density of $1500 \mathrm{~mm}^{-1}$, dispersing the emitted radiation onto a high-resolution CCD camera. The combined energy resolution was $\sim 76.2 \mathrm{meV}$ full width halfmaximum (FWHM), determined by collecting the elastic scattering from a carbon tape reference (see Figure S6). The radiation source is a fixed-gap Apple-II type undulator, ${ }^{37}$ producing left and right circular polarized light as well as linear polarized light. The angular direction of the linear polarization can be varied continuously, from horizontal to vertical with respect to the scattering plane. The XAS spectra were recorded in total electron yield mode.

A permanent gold-coated $\mathrm{NdFeB}$ magnet with a magnetic flux density on the surface of $0.4 \mathrm{~T}$ was used to saturate the magnetization (see Figure S1b). The magnet was placed in two configurations for the measurements: (i) Magnetic circular dichroism configuration where the external magnetic field was aligned parallel to the incident wave vector $\left(\mathbf{k}_{\text {in }}\right)$ (i.e., in the scattering plane) aligned along the [001] crystallographic direction (Figure 1b) and (ii) magnetic linear dichroism configuration where the external magnetic field was aligned perpendicular to $\mathbf{k}_{\text {in }}$ (i.e., out of the scattering plane) aligned along the $[-1 / 2, \sqrt{3} / 2,0]$ crystallographic direction. This low-symmetry direction was chosen to investigate magnetocrystalline anisotropy at the $\mathrm{Fe} \mathrm{A}$ sites of $\mathrm{Fe}_{3} \mathrm{O}_{4}$. All measurements were performed at a fixed temperature of $170 \mathrm{~K}$ (i.e., above the Verwey transition in the cubic phase). To avoid any radiation damage effects, we moved to a fresh spot on the sample every $10 \mathrm{~min}$. During this time period, no signs of radiation damage were detected as shown in Figure S7.

2.3. Calculation Details. We used the quantum many-body program Quanty ${ }^{38-40}$ to simulate Fe XAS and 2p3d RIXS magnetic circular and linear dichroism in $\mathrm{Fe}_{3} \mathrm{O}_{4}$. Three independent cluster calculations were performed to account for the nominal $\mathrm{Fe}^{2+}$ ions in octahedral symmetry $\left(\mathrm{O}_{\mathrm{h}}\right), \mathrm{Fe}^{3+}$ ions in $\mathrm{O}_{\mathrm{h}}$ symmetry, and $\mathrm{Fe}^{3+}$ ions in tetrahedral symmetry $\left(\mathrm{T}_{\mathrm{d}}\right)$. The Hamiltonian used for the calculations consists of the following terms: (i) Coulomb interaction, (ii) crystal field potential, (iii) spin-orbit coupling, and (iv) exchange interaction. The $d-d(p-d)$ multipole part of the Coulomb interaction was scaled to $70 \%$ (80\%) of the Hartree-Fock values of the Slater integral. The general parameters used for the simulations are in agreement with previous studies of $\mathrm{Fe}_{3} \mathrm{O}_{4} L_{2,3}$ edges. ${ }^{41-45}$ All technical details and calculation parameters are reported in the Supporting Information.

We aligned the incident energy of the calculation by fitting the experimental XMCD signal. The relative shift between $\mathrm{Fe}^{3+}$ and $\mathrm{Fe}^{2+}$ $\mathrm{B}$ sites was found to be 0.7 in agreement with previous elaborate investigations. ${ }^{45}$ This value is smaller than the expected chemical shift between $\mathrm{Fe}^{3+}$ and $\mathrm{Fe}^{2+}$ ions in a pure-valence system. This observation is also confirmed by X-ray diffraction measurements where the average bond lengths for both the $\mathrm{Fe}^{3+}$ and $\mathrm{Fe}^{2+}$ ions were found to be very similar in $\mathrm{Fe}_{3} \mathrm{O}_{4}$. ${ }^{15}$

\section{RESULTS AND DISCUSSION}

3.1. Spin-Flip Excitations at the $F e A$ and $B$ Sublattices. The fact that the three $\mathrm{Fe}$ ions in $\mathrm{Fe}_{3} \mathrm{O}_{4}$ contribute to the $L_{2,3}$-edge XAS spectrum renders theoretical calculations necessary to assign spectral features of particular $\mathrm{Fe}$ sites. At an incidence energy of $708.8 \mathrm{eV}$ (labeled $E_{\mathrm{A}}$ ), the resonance is dominantly selective to the $\mathrm{Fe}^{3+} \mathrm{A}$ sites as concluded by theoretical ligand field multiplet calculations (see the right panel of Figure $1 \mathrm{~b}$ ). On the other hand, tuning the incident energy to $E_{\mathrm{B}}=709.1 \mathrm{eV}$ excites mainly the $\mathrm{Fe}^{3+} \mathrm{B}$ sites. $^{41-46}$ The Fe 2p3d RIXS map and high-energy-resolved RIXS slices at incident energies $E_{\mathrm{A}}$ and $E_{\mathrm{B}}$ measurements are shown in Figure 1. A clear energy loss peak is observed at 90 $\mathrm{meV}$ for the RIXS measurement at $E_{\mathrm{A}}$, while only a minor shoulder is observed at $E_{\mathrm{B}}$.

We measured the XMCD signal to provide us with an initial guide for the magnetic contrast. The circular photons carry angular momentum $q=-1 \hbar$ for right circularly polarized light or $q=+1 \hbar$ for left circularly polarized light, which leads to a strong magnetic contrast (dichroism) in a magnetic material as $\mathrm{Fe}_{3} \mathrm{O}_{4}$. Figure 2a shows the XMCD signal together with the theoretical XMCD calculations for the three $\mathrm{Fe}$ ions. The magnetic signal of the $\mathrm{Fe}^{3+} \mathrm{A}$ sites and the $\mathrm{Fe}^{3+} \mathrm{B}$ sites is maximized by tuning the incident energy to $E_{\mathrm{A}}$ and $E_{\mathrm{B}}$. RIXS measurements using circularly polarized $\mathrm{X}$-rays at $E_{\mathrm{A}}\left(E_{\mathrm{B}}\right)$ are presented in Figure 2b (Figure 2c). Energy loss features centered at $\sim 90 \mathrm{meV}$ are now observed for both incident energies (peak fitting is shown in Figures S13 and S14 of the Supporting Information). A strong RIXS-MCD signal is observed for both energies emphasizing the magnetic nature of the feature (see Figure 2d,e). Most importantly, the RIXSMCD signal reverses sign from $E_{\mathrm{A}}$ to $E_{\mathrm{B}}$.

The origin of the sign reversal can be understood by a close inspection of the RIXS process. The RIXS cross section can be 

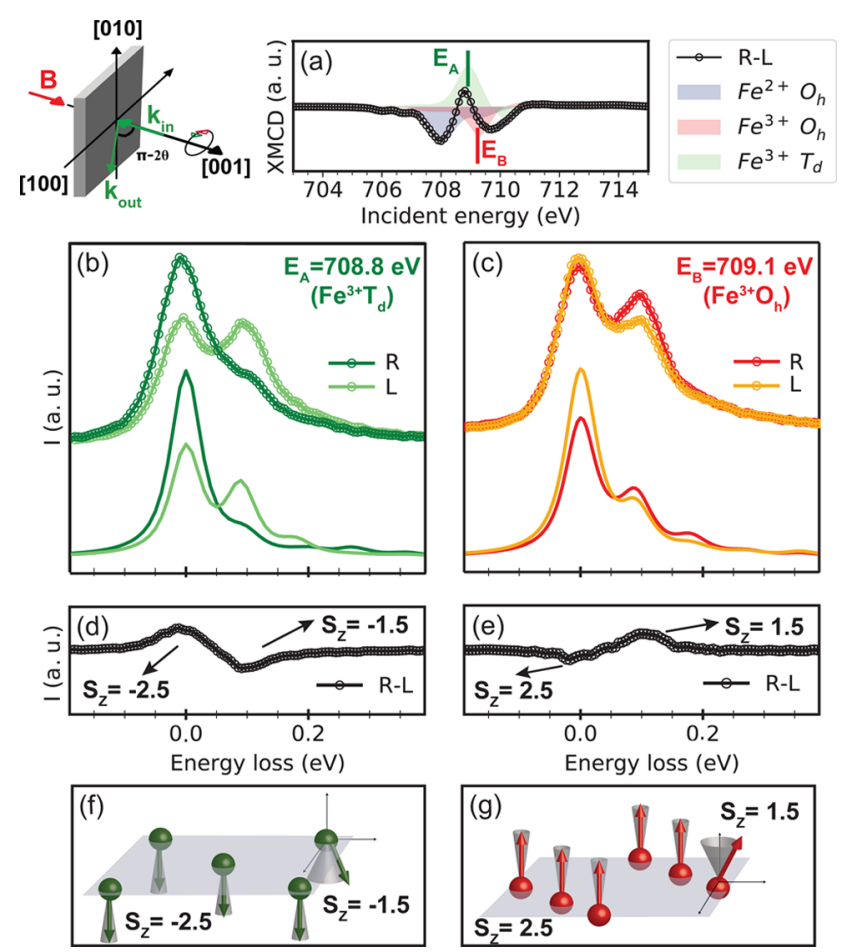

Figure 2. (a) $\mathrm{Fe} L_{3} \mathrm{XMCD}$ signal in $\mathrm{Fe}_{3} \mathrm{O}_{4}$. The contributions of the three $\mathrm{Fe}$ ions to the XMCD signal are shown in filled colors. Highresolution Fe 2p3d experimental RIXS measured at $E_{\mathrm{A}}=708.8 \mathrm{eV}$ and at $E_{\mathrm{B}}=709.1 \mathrm{eV}$ with right $(\mathrm{R})$ and left $(\mathrm{L})$ polarized $\mathrm{X}$-rays are presented in panels (b) and (c), respectively. RIXS simulations performed at the two incident energies are shown in solid lines vertically displaced with respect to the experiment. The experimental RIXS-MCD signals at $E_{\mathrm{A}}$ and at $E_{\mathrm{B}}$ are shown in panels (d) and (e). Schematics of the excitations involved at both incident energies are depicted in panels (f) and (g). At $E_{\mathrm{A}}$ a single spin-flip excitation $\left(\left\langle\Delta S_{z}\right\rangle=1 \hbar\right)$ occurs at the $\mathrm{A}$ sites, while at $E_{\mathrm{B}}$, a single spin-flip excitation $\left(\left\langle\Delta S_{z}\right\rangle=-1 \hbar\right)$ occurs at the B sites.

expressed as a sum of fundamental spectral functions multiplied with a geometry tensor involving the polarization of the incident and scattered photons. ${ }^{47}$ The fundamental spectra can be grouped according to their symmetry into an isotropic contribution, an MCD active contribution, and an MLD active contribution. ${ }^{25}$ Consequently, the 2 p3d RIXSMCD spin-flip excitation cross section is controlled by the $\mathrm{XMCD}$ spectral function, and the sign reversal stems from the incident energy site selectivity. Hence, by measuring Fe 2 p3d RIXS at incident energies $E_{\mathrm{A}}$ and $E_{\mathrm{B}}$, we can directly probe magnetic excitations at the Fe A and B sites separately.

Theoretical calculations with an effective exchange field $J^{*}=$ $90 \mathrm{meV}$ reproduce the experimental data well (Figure 2b,c). Only a minor discrepancy in the intensity between the experimental and simulated elastic peak is observed, which is likely due to saturation and self-absorption effects (refer to the Supporting Information for more details). The $90 \mathrm{meV}$ peak can be assigned to a spin-flip excitation enabled by the core hole spin-orbit coupling in the intermediate state. At $E_{A}$ a single spin-flip excitation $\left(\left\langle\Delta S_{z}\right\rangle=1 \hbar\right)$ at the tetrahedrally coordinated $\mathrm{Fe}^{3+} \mathrm{A}$ site occurs (see Figure $2 \mathrm{f}$ ), while at $E_{\mathrm{B}}$, a single spin-flip excitation $\left(\left\langle\Delta S_{z}\right\rangle=-1 \hbar\right)$ at octahedrally coordinated $\mathrm{Fe}^{3+} \mathrm{B}$ sites occurs (see Figure $2 \mathrm{~g}$ ). The individual contribution of the three Fe ions to the RIXS-MCD signal is shown in SIF:SpinFlipContrib. Our measurements show a shoulder corresponding to a double spin-flip excitation $\left(\left\langle\Delta S_{z}\right\rangle\right.$
$= \pm 2 \hbar$ as shown in Figure S15 of the Supporting Information; however, we do not observe triple or higher-order excitations because the intensities of these peaks are significantly lower. In fact, these higher-order spin-flip excitations $\left(\left\langle\Delta S_{z}\right\rangle= \pm 3 \hbar, 4 \hbar\right.$, and $5 \hbar)$ are possible due to crystal field mixing of the angular momenta as depicited in Figure S16. These excitations are expected to be broad-band-like excitations due to multiple possibilities to propagate the transferred momentum. ${ }^{25,48}$ The effective exchange interactions of the antiferromagnetic coupled A and B sublattices can be extracted from the energy loss peak position and is proportional to the exchange interaction. The energy of the spin-flip excitation at the $\mathrm{Fe}^{3+}$ $\mathrm{B}$ sublattice is roughly related to the exchange interaction according to the relation: $J \approx \frac{90}{12 \times 2.5}=3 \mathrm{meV}$, while the excitation at the $\mathrm{Fe}^{3+}$ A sublattice is roughly related to the exchange interaction as $J \approx \frac{95}{12 \times 4.5}=1.8 \mathrm{meV}$.

We remark that our spin-flip assignment is also consistent with inelastic neutron scattering measurements showing two spin wave modes $\left(\Delta_{5}\right.$ and $\left.\Delta_{2}^{\prime}(\mathrm{B})\right)$ at $\sim 80 \mathrm{meV}$ that nearly do not disperse. ${ }^{49}$ These modes were predicted to propagate solely on the $\mathrm{Fe} B$ sublattice in agreement with our site selectivity at $E_{\mathrm{B}}$. Another mode $\left(\Delta_{2}^{\prime}(\mathrm{A})\right)$ was observed at $\sim 120$ $\mathrm{meV}$, which propagates on the $\mathrm{Fe} A$ sublattice exhibiting only very weak dispersion. We expect to probe this mode by tuning the incident energy to $E_{\mathrm{A}}$; however, our current analysis is not enough to establish the displacement of the peak with $40 \mathrm{meV}$ between the two spin-flip excitations. Although we observe an energy shift between the two spin-flip peaks $(\sim 5 \mathrm{meV}$ as discussed in Table S3 of the Supporting Information), data with better statistics are required to pinpoint the energy position and get accurate fits. In addition, at the resonance of the Fe A sites ( $\mathrm{Fe} \mathrm{B}$ sites), tail excitations from the Fe B sites (Fe A sites) are present leading to the broadening of the spinflip peak in particular given that the energy position of $E_{\mathrm{B}}$ was experimentally underestimated. We have therefore used the same exchange field strength for the Fe A and B sites in our simulations. It is foreseen that with future developments of the energy resolution at RIXS beamlines, it will be possible to disentangle the overlapping peaks as in the case of nickelates. $^{23,27,48,50}$ Nevertheless, the site selectivity of the magnetic excitations in $\mathrm{Fe}_{3} \mathrm{O}_{4}$ is ensured by tuning the incident energy of the RIXS-MCD measurement to couple with magnon modes that are associated with each sublattice separately. As these modes nearly do not disperse for $\mathrm{Fe}_{3} \mathrm{O}_{4}$, they are well explained by our localized multiplet calculations, and the effective exchange field can be extracted from the spin-flip peak. Although phononic excitations were also reported at $\sim 83$ $\mathrm{meV}$, ${ }^{51}$ the strong magnetic circular and linear dichrosim observed together with the excellent agreement of the experimental data with simulations makes them an unlikely assignment.

3.2. Determination of the Orbital Moment through RIXS-MLD. A complementary measurement to undertake is the spin-flip RIXS-MLD measurement, which is sensitive to inplane moments and site anisotropic effects. We focus on the $\mathrm{Fe}^{3+} \mathrm{T}_{\mathrm{d}} \mathrm{A}$ sites in the forthcoming section to illustrate the magnetic contrast attainable from such measurements. The RIXS magnetic linear angular dependence was measured by rotating the incident polarization $\left(\epsilon_{\text {in }}\right)$ with respect to the crystallographic direction and the external magnetic field. We initially measured the RIXS magnetic linear angular dependence on the magnetic field parallel to $\mathbf{k}_{\text {in }}$ (i.e., in the MCD 
configuration) as a reference measurement to check for inplane magnetic contribution. Almost no angular dependence was observed for the spin-flip excitation as can be seen in Figures $\mathrm{S} 8$ and $\mathrm{S} 9$. This is expected for $\mathrm{Fe}_{3} \mathrm{O}_{4}$ because the spin moments are collinear and the magnetization is saturated along the $\mathbf{k}_{\text {in }}$ direction. In this case, the angle between $\epsilon_{\text {in }}$ and the magnetization does not change as a function of the rotation of $\epsilon_{\text {in }}$; only the elastic peak exhibits significant angular dependence due to the zero order Thomson elastic scattering cross section.

The situation changes when the external magnetic field is placed in-plane (the magnetic linear dichroism configuration) as shown in Figure 3a. In this case a strong angular dependence

(a)
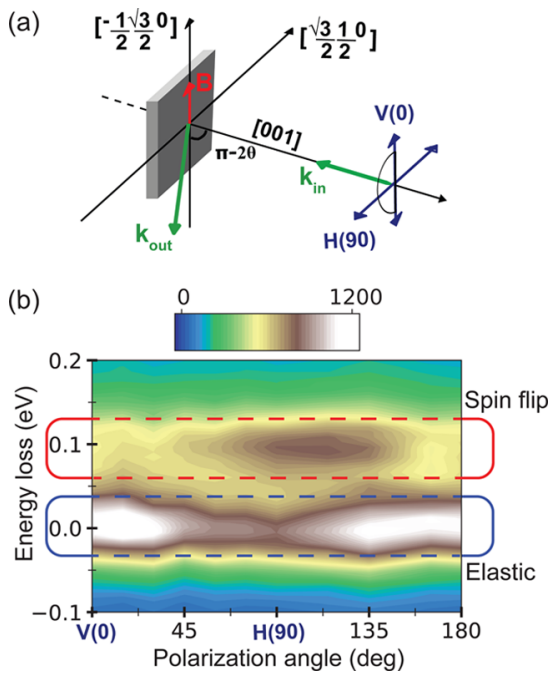

Figure 3. $\mathrm{Fe} 2 \mathrm{p} 3 \mathrm{~d}$ RIXS magnetic linear angular dependence measured at $E_{\mathrm{A}}=708.8 \mathrm{eV}$. (a) Sketch of the setup. (b) Experimental RIXS data measured as a function of the rotation angle of the incident polarization vector. The angle is defined from the vertical direction labeled $V(0)$ and the external magnetic field was aligned parallel to $V(0)$. The elastic and spin-flip features are emphasized with boxes. We note that the map presented is linearly interpolated for visual clarity.

is observed (see Figure $3 \mathrm{~b}$ ) and the angle between $\epsilon_{\text {in }}$ and the magnetization as a function of rotation gives rise to a strong variation at the spin-flip excitation. Theoretical simulations reproduce the angular dependence well and show that the maximum intensity of the spin-flip excitation occurs at $\sim 120^{\circ}$ (Figure 4a). This angle is determined by the orientation of the

(a)

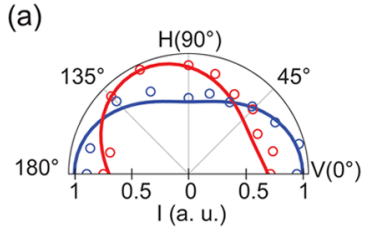

(b)

- Elastic line $(0 \mathrm{meV})$

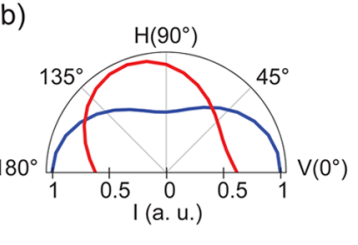

- Spin flip (90 meV)

Figure 4. Fe 2p3d RIXS magnetic linear angular dependence at $E_{\mathrm{A}}=$ 708.8 for the elastic and spin-flip features normalized to the maximum intensity. (a) Experimental data (dotted) for the elastic (blue) and spin-flip excitation (red) are presented in a polar contour plot where the radial axis gives the intensity and the polar axis gives the angle between $\epsilon_{\text {in }}$ and the $V$ axis. A fit of the angular dependence for the two feature with a $\sin ^{2}(\theta)$ function is plotted in solid lines. (b) Calculation of the Fe 2p3d RIXS magnetic linear angular dependence at $E_{\mathrm{A}}$. magnetic moment (with respect to the scattering geometry), and hence a combination of RIXS-MCD and RIXS-MLD measurements can determine the full orientation of the magnetic moment.

Detailed analysis of the RIXS angular dependence can provide more information than just the site-selective exchange interaction and the orientation of the magnetic moment. By aligning the external magnetic field to a low-symmetry direction, we can study site anisotropic effects. Aligning the external magnetic field to a low crystallographic symmetry direction aligns the net spin moment parallel to the magnetic field. If the orbital moment is not fully quenched (because of charge transfer or distortion), it consequently also realigns toward the low-symmetry direction. The final orientation of the net magnetic moment depends on the strength of the competing interactions such as spin-orbit coupling, exchange, and distortion. Hence, the phase shift of the maximum intensity of the spin-flip excitation can be used to quantify magnetic-moment-induced distortion of the electron cloud. Based on this concept, we explored the possibility of having unquenched orbital moment at the $\mathrm{Fe} A$ sites. Nominal $\mathrm{Fe}^{3+}$ $T_{d}$ ions should have no orbital moment (high-spin $3 \mathrm{~d}^{5}$ ion); however, it has been reported that a strong non-vanishing orbital moment is possibly present at the $\mathrm{Fe} \mathrm{A}$ sites as a result of the charge transfer from the neighboring oxygens. ${ }^{52}$ Our measurements show that the maximum of the spin-flip angular dependence occurs at $\sim 120^{\circ}$. This angle agrees well with the result of our calculation and corresponds purely to the sample displacement $\left(90^{\circ}+30^{\circ}\right)$. The angular dependence follows that expected for the case of $\mathbf{l}_{z} \approx 0$ (compare the experiment to the calculations in Figure 4a,b).

3.3. Charge Transfer Effects. It is of conceptual interest to investigate the sensitivity of the RIXS-MLD signal at spinflip excitations to charge transfer and orbital polarization effects. At oxide interfaces, charge transfer can be driven by a difference in chemical potential. In addition, the symmetry lowering frequently induces interfacial orbital angular momentum, ${ }^{53}$ which in turn modifies the magnetic properties. In such scenarios, it is not only interesting to determine the orientation of the magnetic moment but also the orbital contribution to it. Only a few techniques can distinguish between the magnetic orbital and spin moment contributions. A particular challenge is encountered when the popular XMCD technique fails for antiferromagnetic systems and non-trivial spin textures. We investigated through theoretical simulations the effect of a small hybridization potential between the ligands (surrounding oxygen anions) and the $\mathrm{Fe}^{3+} \mathrm{T}_{\mathrm{d}}$ cation on the angular dependence of the spin-flip excitation. Two different hybridization types were studied, namely, $\pi$ - and $\sigma$-type hybridization potentials (see Figure $5 b, c)$.

We started by assuming that the effective exchange interaction is not affected by the hybridization. This is a reasonable assumption because only small changes in the spin angular momentum were found for calculations performed using realistic hybridization potentials. Typically, a reduction of $\left\langle S^{2}\right\rangle$ from $8.75 \hbar^{2}$ to $8.74 \hbar^{2}$ was predicted by our calculations. Therefore, it can be concluded that the effective exchange field does not significantly change and that the spinflip excitation will appear at the same (or very close) energy position to the previous case. The charge transfer from the ligands to the metal induces an orbital angular momentum at the metal site. Although the charge transfer is small, its effect 


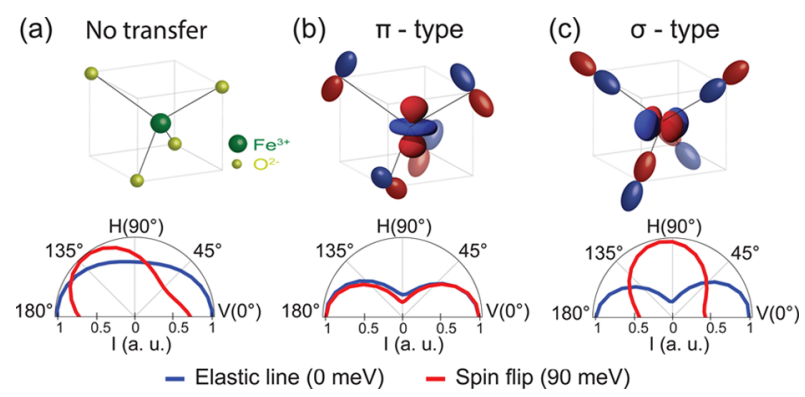

Figure 5. Calculated angular dependence of the elastic and spin-flip excitation normalized to the maximum intensity for the following three cases. (a) Crystal field calculation where no charge transfer between the oxygen and $\mathrm{Fe}^{3+}$ ion occurs. (b) Charge transfer is allowed for the $d_{z}{ }^{2}$ orbital, that is, $\pi$-type. (c) Charge transfer is allowed for the $\mathrm{d}_{x y}$ orbital, that is, $\sigma$-type.

can potentially be large depending on the symmetry of the hybridization (i.e., depending on which orbitals the partially transferred electrons reside). We found a change from $\left\langle L^{2}\right\rangle=0$ to $\left\langle L^{2}\right\rangle=3.17 \hbar^{2}$ and $\left\langle L^{2}\right\rangle=1.06 \hbar^{2}$ for $\pi$ - (Figure $5 b$ ) and $\sigma$ type (Figure 5c) channels, respectively.

The change of the ground-state symmetry as a result of the charge transfer has visible effects on the angular dependence of the spin-flip excitation (compare the angular dependence in Figure $5 \mathrm{a}-\mathrm{c}$ ). Although in the three cases, the two-fold symmetry of the angular dependence is preserved, the angle at which the maximum intensity of the spin-flip excitation shows significant changes. Hence, the presence of an orbital contribution to the magnetic moment can be concluded when a phase shift of the spin-flip excitation is observed. Through comparing the phase shift to simulations, the magnetic orbital moment can be quantified.

\section{CONCLUSIONS}

In conclusion, we demonstrated that the magnetic contrast obtained at spin-flip excitations is an element and site-selective direct probe of the magnetic ordering and the exchange interaction. Because RIXS is sensitive to the orbital degree of freedom, a thorough analysis of the angular dependence at spin-flip excitations can be used to identify the orbital polarization. The combination of RIXS-MCD and RIXSMLD measurements at spin-flip excitations represent an important methodological step forward as they permit the study of magnetism in thin films and interfaces where many interesting spin textures emerge. The potential to use this magnetic contrast in an RIXS microscopy setup with nanometer resolution ${ }^{54}$ will open the realms of possibilities for investigating engineered magnetization. We expect that such mapping experiments will be possible in the near future with the upgrade of synchrotron sources to diffraction limited storage rings.

\section{ASSOCIATED CONTENT}

\section{S Supporting Information}

The Supporting Information is available free of charge on the ACS Publications website at DOI: 10.1021/acsami.9b10196.

Sample preparation and characterization, RIXS measurements, calculations, spin-flip peak fitting, higher-order spin-flip excitations, and saturation and self-absorption effects (PDF)

\section{AUTHOR INFORMATION}

Corresponding Authors

*E-mail: H.M.E.A.Elnaggar@uu.nl (H.E.).

*E-mail: F.M.F.deGroot@uu.nl (F.M.F.d.G.).

ORCID

Hebatalla Elnaggar: 0000-0002-4223-4054

Ru-Pan Wang: 0000-0003-4495-9881

Frank M. F. de Groot: 0000-0002-1340-2186

Notes

The authors declare no competing financial interest.

\section{ACKNOWLEDGMENTS}

We thank H. Guo, A. Bosak, F. Frati, and M. Hussein for their help with cutting the sample, performing X-ray diffraction measurements, and sample characterization. We are grateful for the fruitful discussions with A. Juhin, Ch. Brouder, M.-A. Arrio, Ph. Sainctavit, and H. Tjeng. We thank Y. Uemura, F. Meirer, $\mathrm{K}$. Kvashnina, and O. Elsharkasy for thoroughly reading the manuscript and their feedback. We acknowledge the support of the Laue lab at the Paul Scherrer Institut (PSI). We thank Thomas Schmidt for his valuable assistance with the experiment. The synchrotron experiments have been performed at the ADRESS beamline of the Swiss Light Source (SLS) at the PSI. The work at PSI is supported by the Swiss National Science Foundation through the NCCR MARVEL and the Sinergia network Mott Physics Beyond the Heisenberg Model (MPBH). M.S. acknowledges support from the National Science Center of Poland (2014/14/E/ST3/ 00026). This work was financed by the ERC advanced Grant XRAYonACTIVE no. 340279.

\section{REFERENCES}

(1) Al Ma'Mari, F.; Rogers, M.; Alghamdi, S.; Moorsom, T.; Lee, S.; Prokscha, T.; Luetkens, H.; Valvidares, M.; Teobaldi, G.; Flokstra, M.; Stewart, R.; Gargiani, P.; Ali, M.; Burnell, G.; Hickey, B. J.; Cespedes, $\mathrm{O}$. Emergent Magnetism at transition-metal-nanocarbon Interfaces. PNAS 2017, 114, 5583-5588.

(2) Meckler, S.; Mikuszeit, N.; Preßler, A.; Vedmedenko, E. Y.; Pietzsch, O.; Wiesendanger, R. Real-Space Observation of a RightRotating Inhomogeneous Cycloidal Spin Spiral by Spin-Polarized Scanning Tunneling Microscopy in a Triple Axes Vector Magnet. Phys. Rev. Lett. 2009, 103, 157201.

(3) Heinze, S.; von Bergmann, K.; Menzel, M.; Brede, J.; Kubetzka, A.; Wiesendanger, R.; Bihlmayer, G.; Blügel, S. Spontaneous AtomicScale Magnetic Skyrmion Lattice in two Dimensions. Nat. Phys. 2011, 7,713 .

(4) Romming, N.; Hanneken, C.; Menzel, M.; Bickel, J. E.; Wolter, B.; von Bergmann, K.; Kubetzka, A.; Wiesendanger, R. Writing and Deleting Single Magnetic Skyrmions. Science 2013, 341, 636-639.

(5) Romming, N.; Kubetzka, A.; Hanneken, C.; von Bergmann, K.; Wiesendanger, R. Field-Dependent Size and Shape of Single Magnetic Skyrmions. Phys. Rev. Lett. 2015, 114, 177203.

(6) Liao, Z.-M.; Li, Y.-D.; Xu, J.; Zhang, J.-M.; Xia, K.; Yu, D.-P. Spin-Filter Effect in Magnetite Nanowire. Nano Lett. 2006, 6, 10871091.

(7) Ramos, R.; Kikkawa, T.; Uchida, K.; Adachi, H.; Lucas, I.; Aguirre, M. H.; Algarabel, P.; Morellón, L.; Maekawa, S.; Saitoh, E.; Ibarra, M. R. Observation of the Spin Seebeck Effect in Epitaxial $\mathrm{Fe}_{3} \mathrm{O}_{4}$ Thin Films. Appl. Phys. Lett. 2013, 102, No. 072413.

(8) Pontius, N.; Kachel, T.; Schüßler-Langeheine, C.; Schlotter, W. F.; Beye, M.; Sorgenfrei, F.; Chang, C. F.; Föhlisch, A.; Wurth, W.; Metcalf, P.; Leonov, I.; Yaresko, A.; Stojanovic, N.; Berglund, M.; Guerassimova, N.; Düsterer, S.; Redlin, H.; Dürr, H. A. TimeResolved Resonant Soft X-ray Diffraction with Free-Electron Lasers: 
Femtosecond Dynamics across the Verwey Transition in Magnetite. Appl. Phys. Lett. 2011, 98, 182504.

(9) de Jong, S.; Kukreja, R.; Trabant, C.; Pontius, N.; Chang, C. F.; Kachel, T.; Beye, M.; Sorgenfrei, F.; Back, C. H.; Bräuer, B.; Schlotter, W. F.; Turner, J. J.; Krupin, O.; Doehler, M.; Zhu, D.; Hossain, M. A.; Scherz, A. O.; Fausti, D.; Novelli, F.; Esposito, M.; Lee, W. S.; Chuang, Y. D.; Lu, D. H.; Moore, R. G.; Yi, M.; Trigo, M.; Kirchmann, P.; Pathey, L.; Golden, M. S.; Buchholz, M.; Metcalf, P.; Parmigiani, F.; Wurth, W.; Föhlisch, A.; Schüßler-Langeheine, C.; Dür, H. A. Speed Limit of the Insulator-Metal Transition in Magnetite. Nat. Mater. 2013, 12, 882.

(10) Verwey, E. J. W. Electronic Conduction of Magnetite $\left(\mathrm{Fe}_{3} \mathrm{O}_{4}\right)$ and its Transition Point at Low Temperatures. Nature 1939, 144, 327-328.

(11) Walz, F. The Verwey Transition - A Topical Review. J. Phys.: Condens. Matter 2002, 14, R285.

(12) Subías, G.; García, J.; Proietti, M. G.; Blasco, J.; Renevier, H.; Hodeau, J. L.; Sánchez, M. C. X-ray Resonant Scattering of $(004 n+2)$ Forbidden Reflections in Spinel Ferrites. Phys. Rev. B 2004, 70, 155105.

(13) Subías, G.; García, J.; Blasco, J. EXAFS Spectroscopic Analysis of the Verwey Transition in $\mathrm{Fe}_{3} \mathrm{O}_{4}$. Phys. Rev. B 2005, 71, 155103.

(14) Uzu, H.; Tanaka, A. Charge and Complex Orbital Ordering in $\mathrm{Fe}_{3} \mathrm{O}_{4}$. Phys. B 2006, 378-380, 571-573.

(15) Senn, M. S.; Wright, J. P.; Attfield, J. P. Charge Order and Three-Site Distortions in the Verwey Structure of Magnetite. Nature 2012, 481, 173-176.

(16) Tanaka, A.; Chang, C. F.; Buchholz, M.; Trabant, C.; Schierle, E.; Schlappa, J.; Schmitz, D.; Ott, H.; Metcalf, P.; Tjeng, L. H.; Schüßler-Langeheine, C. Analysis of Charge and Orbital Order in $\mathrm{Fe}_{3} \mathrm{O}_{4}$ by $\mathrm{Fe} \mathrm{L}_{2,3}$ Resonant X-ray Diffraction. Phys. Rev. B 2013, 88, 195110.

(17) Hoesch, M.; Piekarz, P.; Bosak, A.; Le Tacon, M.; Krisch, M.; Kozłowski, A.; Oleś, A. M.; Parlinski, K. Anharmonicity due to Electron-Phonon Coupling in Magnetite. Phys. Rev. Lett. 2013, 110, 207204.

(18) Bosak, A.; Chernyshov, D.; Hoesch, M.; Piekarz, P.; Le Tacon, M.; Krisch, M.; Kozlowski, A.; Oleś, A. M.; Parlinski, K. Short-Range Correlations in Magnetite above the Verwey Temperature. Phys. Rev. $X$ 2014, 4, 011040.

(19) Hodeau, J.-L.; Favre-Nicolin, V.; Bos, S.; Renevier, H.; Lorenzo, E.; Berar, J.-F. Resonant Diffraction. Chem. Rev. 2001, 101, 18431868.

(20) Haverkort, M. W.; Hollmann, N.; Krug, I. P.; Tanaka, A. Symmetry Analysis of Magneto-Optical Effects: The Case of X-ray Diffraction and X-ray Absorption at the Transition metalL2,3edge. Phys. Rev. B 2010, 82, No. 094403.

(21) Ament, L. J. P.; van Veenendaal, M.; Devereaux, T. P.; Hill, J. P.; van den Brink, J. Resonant Inelastic X-ray Scattering studies of Elementary Excitations. Rev. Mod. Phys. 2011, 83, 705-767.

(22) Hancock, J. N.; Jarrige, I. The Promise of Resonant Inelastic Xray Scattering for Modern Kondo Physics. J. Magn. Magn. Mater. 2016, 400, 41-46.

(23) De Groot, F. M. F.; Kuiper, P.; Sawatzky, G. A. Local spin-flip spectral Distribution Obtained by Resonant X-ray Raman Scattering. Phys. Rev. B 1998, 57, 14584-14587.

(24) van Veenendaal, M. Polarization Dependence of L- and MEdge Resonant Inelastic X-ray Scattering in Transition-Metal Compounds. Phys. Rev. Lett. 2006, 96, 117404.

(25) Haverkort, M. W. Theory of Resonant Inelastic X-ray Scattering by Collective Magnetic Excitations. Phys. Rev. Lett. 2010, $105,167404$.

(26) Jia, C.; Wohlfeld, K.; Wang, Y.; Moritz, B.; Devereaux, T. P. Using RIXS to Uncover Elementary Charge and Spin Excitations. Phys. Rev. X 2016, 6, 021020.

(27) Lu, Y.; Betto, D.; Fürsich, K.; Suzuki, H.; Kim, H.-H.; Cristiani, G.; Logvenov, G.; Brookes, N. B.; Benckiser, E.; Haverkort, M. W.; Khaliullin, G.; Le Tacon, M.; Minola, M.; Keimer, B. Site-selective
Probe of Magnetic Excitations in Rare-Earth Nickelates using Resonant Inelastic X-ray Scattering. Phys. Rev. X 2018, 8, 031014.

(28) Ament, L. J. P.; Ghiringhelli, G.; Sala, M. M.; Braicovich, L.; van den Brink, J. Theoretical Demonstration of How the Dispersion of Magnetic Excitations in Cuprate Compounds can be Determined Using Resonant Inelastic X-ray Scattering. Phys. Rev. Lett. 2009, 103, 117003.

(29) Braicovich, L.; Ament, L. J. P.; Bisogni, V.; Forte, F.; Aruta, C.; Balestrino, G.; Brookes, N. B.; De Luca, G. M.; Medaglia, P. G.; Granozio, F. M.; Radovic, M.; Salluzzo, M.; van den Brink, J.; Ghiringhelli, G. Dispersion of Magnetic Excitations in the Cuprate $\mathrm{La}_{2} \mathrm{CuO}_{4}$ and $\mathrm{CaCuO}_{2}$ Compounds Measured Using Resonant X-ray Scattering. Phys. Rev. Lett. 2009, 102, 167401.

(30) Schlappa, J.; Schmitt, T.; Vernay, F.; Strocov, V. N.; Ilakovac, V.; Thielemann, B.; Rønnow, H. M.; Vanishri, S.; Piazzalunga, A.; Wang, X.; Braicovich, L.; Ghiringhelli, G.; Marin, C.; Mesot, J.; Delley, B.; Patthey, L. Collective Magnetic Excitations in the Spin Ladder $\mathrm{Sr}_{14} \mathrm{Cu}_{24} \mathrm{O}_{41}$ Measured using High-Resolution Resonant Inelastic X-ray Scattering. Phys. Rev. Lett. 2009, 103, 047401.

(31) Braicovich, L.; van den Brink, J.; Bisogni, V.; Sala, M. M.; Ament, L. J. P.; Brookes, N. B.; De Luca, G. M.; Salluzzo, M.; Schmitt, T.; Strocov, V. N.; Ghiringhelli, G. Magnetic Excitations and Phase Separation in the Underdoped $\mathrm{La}_{2-\mathrm{x}} \mathrm{Sr}_{\mathrm{x}} \mathrm{CuO}_{4}$ Superconductor Measured by Resonant Inelastic X-ray Scattering. Phys. Rev. Lett. 2010, 104, 077002.

(32) Braicovich, L.; van der Laan, G.; Ghiringhelli, G.; Tagliaferri, A.; van Veenendaal, M. A.; Brookes, N. B.; Chervinskii, M. M.; Dallera, C.; De Michelis, B.; Dürr, H. A. Magnetic Circular Dichroism in Resonant Raman Scattering in the Perpendicular Geometry at the L edge of 3d Transition Metal Systems. Phys. Rev. Lett. 1999, 82, 15661569.

(33) van der Laan, G.; Ghiringhelli, G.; Tagliaferri, A.; Brookes, N. B.; Braicovich, L. Quenching of Atomiclike Properties upon SolidState Formation: Quantitative Comparison between $\mathrm{Co}$ and $\mathrm{Ni}$ in Ferrites studied by X-ray Resonant Raman Scattering at the $\mathrm{L}_{3}$ edge. Phys. Rev. B 2004, 69, 104427.

(34) Braicovich, L.; van der Laan, G.; Tagliaferri, A.; Annese, E.; Ghiringhelli, G.; Brookes, N. B. Resonant Inelastic X-ray Scattering from Magnetic Systems with Angular Resolution and Polarization Analysis of the Scattered Beam: Results on Metallic Co, Fe, and Co Ferrite at the $\mathrm{L}_{3,2}$ edges. Phys. Rev. B 2007, 75, 184408.

(35) Fumagalli, R.; Braicovich, L.; Minola, M.; Peng, Y.; Kummer, K.; Betto, D.; Rossi, M.; Lefrancois, E.; Morawe, C.; Salluzzo, M.; Suzuki, H.; Yakhou, F.; Le Tacon, M.; Keimer, B.; Brookes, N.; Moretti Sala, M.; Ghiringhelli, G. Polarization Resolved $\mathrm{Cu} \mathrm{L}_{3}$-edge Resonant Inelastic X-ray Scattering of Orbital and Spin Excitations in $\mathrm{NdBa}_{2} \mathrm{Cu}_{3} \mathrm{O}_{7-\delta}$. arXiv 2019, 1902.05471 [cond-mat.supr-con].

(36) Strocov, V. N.; Schmitt, T.; Flechsig, U.; Schmidt, T.; Imhof, A.; Chen, Q.; Raabe, J.; Betemps, R.; Zimoch, D.; Krempasky, J.; Wang, X.; Grioni, M.; Piazzalunga, A.; Patthey, L. High-Resolution Soft X-ray beamline ADRESS at the Swiss Light Source for Resonant Inelastic X-ray Scattering and Angle-Resolved Photoelectron Spectroscopies. J. Synchrotron Radiat. 2010, 17, 631-643.

(37) Sasaki, S.; Kakuno, K.; Takada, T.; Shimada, T.; ichi Yanagida, K.; Miyahara, Y. Design of a New Type of Planar Undulator for Generating Variably Polarized Radiation. Nucl. Instrum. Methods Phys. Res., Sect. A 1993, 331, 763-767.

(38) Haverkort, M. W.; Zwierzycki, M.; Andersen, O. K. Multiplet Ligand-Field Theory using Wannier Orbitals. Phys. Rev. B 2012, 85, 165113.

(39) Lu, Y.; Höppner, M.; Gunnarsson, O.; Haverkort, M. W. Efficient Real-Frequency Solver for Dynamical Mean-Field Theory. Phys. Rev. B 2014, 90, 085102.

(40) Haverkort, M. W.; Sangiovanni, G.; Hansmann, P.; Toschi, A.; Lu, Y.; Macke, S. Bands, Resonances, Edge Singularities and Excitons in Core Level Spectroscopy Investigated within the Dynamical MeanField theory. EPL 2014, 108, 57004. 
(41) Kuiper, P.; Searle, B. G.; Duda, L. C.; Wolf, R. M.; van der Zaag, P. J. Fe $\mathrm{L}_{2,3}$ Linear and Circular Magnetic Dichroism of $\mathrm{Fe}_{3} \mathrm{O}_{4}$. J. Electron Spectrosc. Relat. Phenom. 1997, 86, 107-113.

(42) Pattrick, R. A. D.; van der Laan, G.; Henderson, C. M. B.; Kuiper, P.; Dudzik, E.; Vaughan, D. J. Cation Site Occupancy in Spinel Ferrites Studied by X-ray Magnetic Circular Dichroism: Developing a Method for Mineralogists. Eur. J. Mineral. 2002, 14, 1095-1102.

(43) Arenholz, E.; van der Laan, G.; Chopdekar, R. V.; Suzuki, Y. Anisotropic X-ray Magnetic Linear Dichroism at the $\mathrm{Fe} \mathrm{L}_{2,3}$ Edges in $\mathrm{Fe}_{3} \mathrm{O}_{4}$. Phys. Rev. B 2006, 74, 094407.

(44) Carvallo, C.; Sainctavit, P.; Arrio, M. A.; Menguy, N.; Wang, Y.; Ona-Nguema, G.; Brice-Profeta, S. Biogenic vs. Abiogenic Magnetite Nanoparticles: A XMCD Study. Am. Mineral. 2008, 93, 880-885.

(45) Liu, B.; Piamonteze, C.; Delgado-Jaime, M. U.; Wang, R.-P.; Heidler, J.; Dreiser, J.; Chopdekar, R.; Nolting, F.; de Groot, F. M. F. Sum Rule Distortions in fluorescence-yield X-ray Magnetic Circular Dichroism. Phys. Rev. B 2017, 96, 054446.

(46) Chang, C. F.; Hu, Z.; Klein, S.; Liu, X. H.; Sutarto, R.; Tanaka, A.; Cezar, J. C.; Brookes, N. B.; Lin, H.-J.; Hsieh, H. H.; Chen, C. T.; Rata, A. D.; Tjeng, L. H. Dynamic Atomic Reconstruction: How $\mathrm{Fe}_{3} \mathrm{O}_{4}$ Thin Films Evade Polar Catastrophe for Epitaxy. Phys. Rev. X 2016, 6, 041011.

(47) Juhin, A.; Brouder, C.; Groot, F. Angular Dependence of Resonant Inelastic x-ray Scattering: A Spherical Tensor Expansion. Open Phys. 2014, 12, 323-340.

(48) Betto, D.; Peng, Y. Y.; Porter, S. B.; Berti, G.; Calloni, A.; Ghiringhelli, G.; Brookes, N. B. Three-dimensional Dispersion of Spin Waves Measured in $\mathrm{NiO}$ by Resonant Inelastic X-ray Scattering. Phys. Rev. B 2017, 96, 020409.

(49) McQueeney, R. J.; Yethiraj, M.; Chang, S.; Montfrooij, W.; Perring, T. G.; Honig, J. M.; Metcalf, P. Zener Double Exchange from Local Valence Fluctuations in Magnetite. Phys. Rev. Lett. 2007, 99, 246401.

(50) Ghiringhelli, G.; Piazzalunga, A.; Dallera, C.; Schmitt, T.; Strocov, V. N.; Schlappa, J.; Patthey, L.; Wang, X.; Berger, H.; Grioni, M. Observation of two Nondispersive Magnetic Excitations in $\mathrm{NiO}$ by Resonant Inelastic Soft-X-Ray Scattering. Phys. Rev. Lett. 2009, 102, 027401 .

(51) Gasparov, L. V.; Tanner, D. B.; Romero, D. B.; Berger, H.; Margaritondo, G.; Forro, L. Infrared and Raman studies of the Verwey Transition in Magnetite. Phys. Rev. B 2000, 62, 7939-7944.

(52) Goering, E. Large Hidden Orbital Moments in Magnetite. Phys. Status Solidi B 2011, 248, 2345-2351.

(53) Hellman, F.; Hoffmann, A.; Tserkovnyak, Y.; Beach, G. S. D.; Fullerton, E. E.; Leighton, C.; MacDonald, A. H.; Ralph, D. C.; Arena, D. A.; Dürr, H. A.; Fischer, P.; Grollier, J.; Heremans, J. P.; Jungwirth, T.; Kimel, A. V.; Koopmans, B.; Krivorotov, I. N.; May, S. J.; PetfordLong, A. K.; Rondinelli, J. M.; Samarth, N.; Schuller, I. K.; Slavin, A. N.; Stiles, M. D.; Tchernyshyov, O.; Thiaville, A.; Zink, B. L. Interface-Induced Phenomena in Magnetism. Rev. Mod. Phys. 2017, 89, 025006.

(54) Marschall, F.; McNally, D.; Guzenko, V. A.; Rösner, B.; Dantz, M.; Lu, X.; Nue, L.; Strocov, V.; Schmitt, T.; David, C. Zone Plates as Imaging Analyzers for Resonant Inelastic X-ray Scattering. Opt. Express 2017, 25, 15624-15634.

\section{NOTE ADDED AFTER ASAP PUBLICATION}

This paper was published ASAP on September 20, 2019, with errors in the Abstract, Figure 2 caption, and the Supporting Information. The corrected version was reposted on September 24, 2019. 